\title{
SEE yield, $\delta$, and image contrast in LVSEM : some new correlations ?
}

\author{
J. Cazaux
}

\author{
DTI, CNRS UMR 6107,UFR Sciences, BP 1039, 51687 Reims Cedex 2, France.
}

Many causes (topographic, chemical, particle, charging) of image contrast in SEM have been identified from a long time [1]. From some recent developments in the physics of the Secondary Electron Emission (SEE) from insulators, some other possible causes are suggested here.

Crystallite size contrast. Years ago, Ushio et al, reported on the significant difference in the SEE yield, $\delta$, of different $\mathrm{MgO}$ films [2]. The application of a recent random walk model to the transport of the SEE including their reflection on the grain boundaries reinforces the idea on the possible role of the crystallitesize effect on the secondary electron yield $\delta$ from insulators [3 and Fig.1]. The experimental evidence would consist in the SEM imaging of a sintered powder of an insulating material with two different grainsize-regions in the common field of view. At $\mathrm{E}_{0}>3 \mathrm{keV}$ and with a E-T detector, the region having larger grains would appear brighter than the other. Thin sintered powders ( $\mathrm{D}$ a few $\mathrm{nm}$ ) backed by a metal must be used to prevent charging.

Thickness contrast. As shown on Fig 2 for solid Ar films condensed on a $\mathrm{Cu}$ substrate, the yield $\delta$ of coated specimens changes with the thickness of the coating, D. When D increases, the initial step may correspond to a significant change of the yield associated to only a slight change of the surface composition of the substrate material because SEE escape probability may change deeply with the surface affinity, $\chi$. Next, there is the electron emission from the film added to that from the substrate (when $\mathrm{D}<\mathrm{s}$; s: escape depth of the SEs) and one difficulty is to evaluate the transmission probability of the SEs at the coating/substrate interface. Next, in the intermediate case, $\mathrm{s}<\mathrm{D}<<\mathrm{R}$, the SEE yield is restricted to the coating but its $\mathrm{SE}_{2}$ contribution is influenced by the backscattering coefficient of the substrate, $\eta_{\mathrm{S}}: \delta_{\mathrm{B}}=\mathrm{k}$ $\eta_{S} \delta_{P}(1)$. At end, the yield of thick coating is expected to be that of the coating material because all the PEs are confined in the film material (D>>R). In fact, Fig. 2 shows that $\delta$ may not saturate even when the $\mathrm{D}$ value largely exceeds the $\mathrm{R}$ value. This surprising fact may be due to a possible change of the work function with thickness and charging. The complexity of the full evolution of $\delta$ explains the lack of general theory for the SEE yield from stratified materials but it does not forbid to explain some thickness contrasts such as that shown on Fig 3. The image on the left shows an example of the frequently reported carbon contamination contrast. It is typically a thickness contrast obtained, here, on diamond substrate (same $\mathrm{Z}$ for the film and the substrate). The image on the right illustrates the effect of holes in the (Ag) substrate on the local value of $\delta_{\mathrm{B}}$ and then on the contrast of the image. In many situations, the interpretation of the observed contrast is facilitated by correlating $\delta$ ( $\mathrm{E} T$ and in-lens detectors) images to $\eta$ image, in particular, with the use of the scatter diagram technique ([4] and work in progress).

References

[1] C.E Lyman et al, Scanning Electron Microsc., a laboratory workbook, Plenum, NY. 1990

[2] Y. Ushio et al, Thin Solid Films, 167, (1988) 299

[3] J. Cazaux , Thin Solid Films, in press.

[4] J. M. Patat et al, Scanning, 24 (2002) 109 

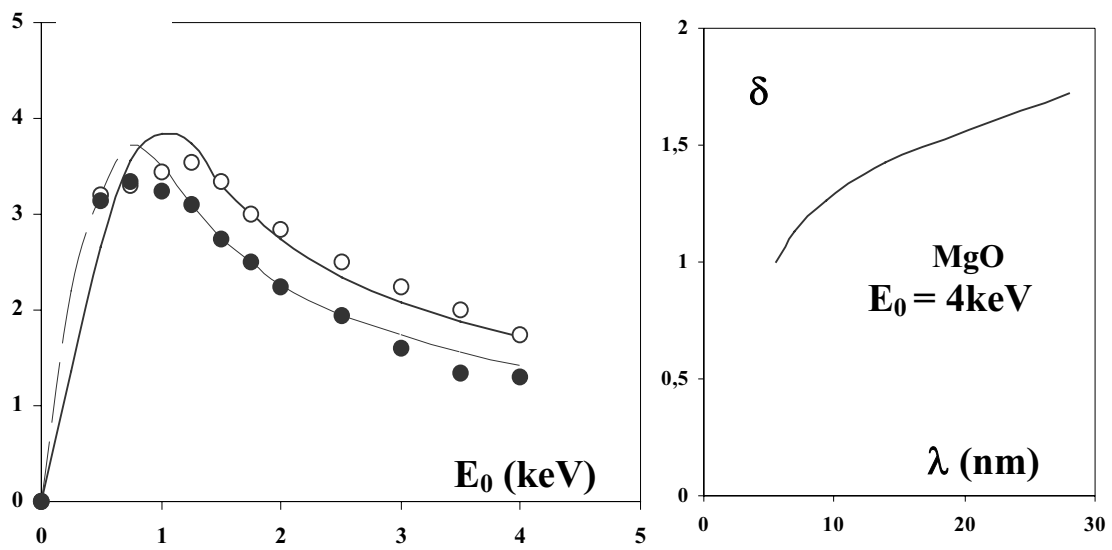

Fig 1. SEE yield from $\mathrm{MgO}$. Left: Data obtained from various $\mathrm{MgO}$ films (symbols;[2]), compared to curves deduced from a model including the scattering of the SE's. Their attenuation length decreases like $\mathrm{n}^{-1 / 2}$ (n:number of scattering events) for the same maximum path $\left(\mathrm{r} \sim 28 \mathrm{~nm}\right.$ ). Right: At $\mathrm{E}_{0}=4 \mathrm{keV}$ expected change of $\delta$ as a function of the grain size (or of the transport mean free path, $\lambda$ ) [3].

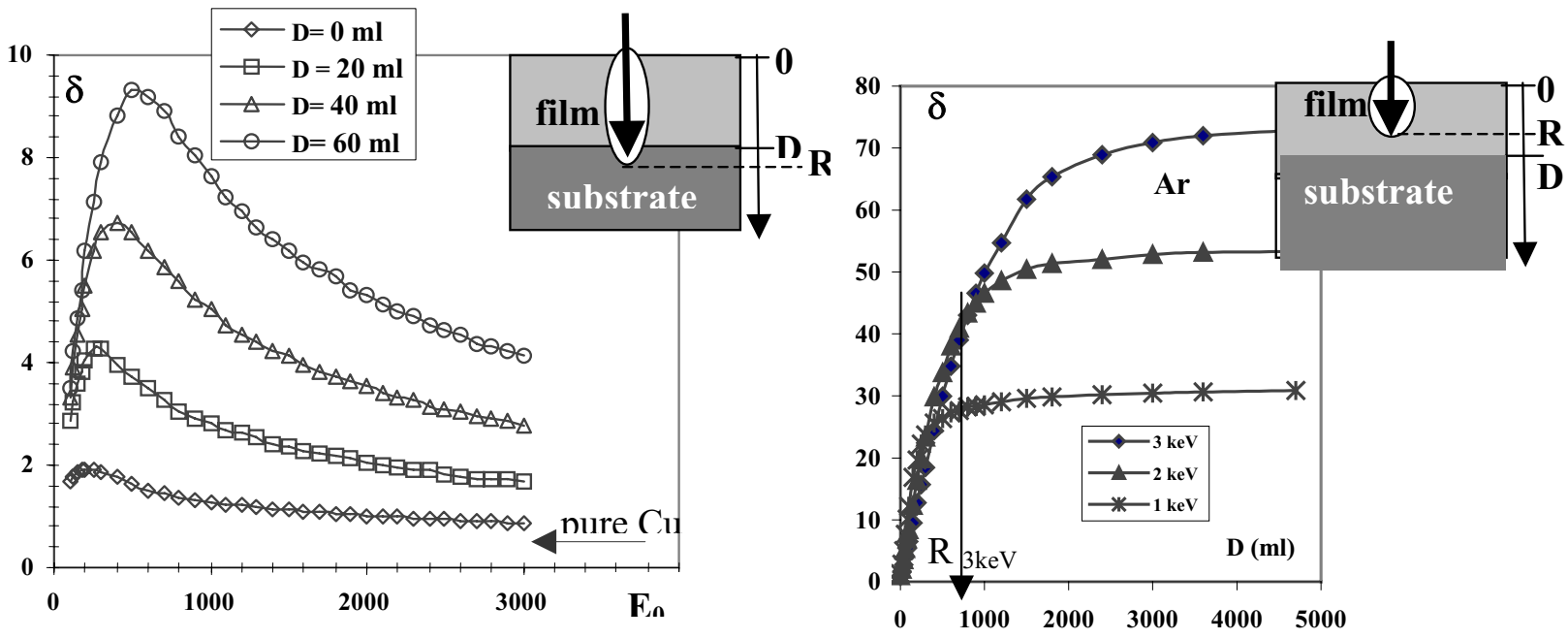

Fig. 2. Left. SEE yield, $\delta$, (vs beam energy, $\mathrm{E}_{0}$ ) of Ar films of various thickness deposited on a copper substrate: $\mathrm{D}(10 \mathrm{ml})=2.66 \mathrm{~nm}$. See the change of $\delta$ with $\mathrm{D}$. Right. Change of $\delta$ vs $\mathrm{D}$ for various $\mathrm{E}_{0}$ values. Note the increase of $\delta$ above the calculated range, $\mathrm{R}_{3 \mathrm{keV}}$, of the PEs . Similar results also hold for $\mathrm{Ne} ; \mathrm{Kr}$ and $\mathrm{Xe}$ (work in cooperation with N. Hillaret, CERN, CH).
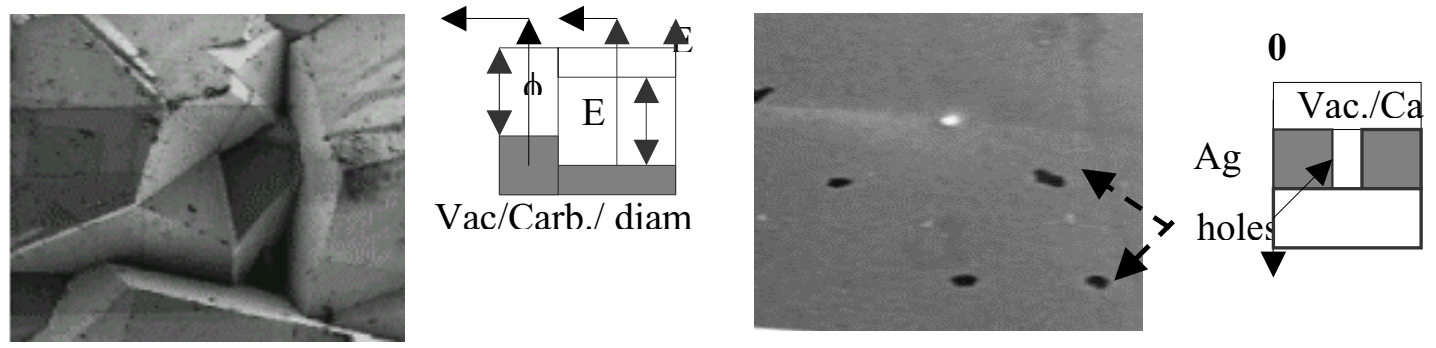

Fig 3. Examples of thickness contrast in SEM imaging. Left $\left(\mathrm{E}_{0}=1 \mathrm{keV}\right)$ : Carbon contamination layer on a diamond surface (with its associated band struct.). Right $\left(\mathrm{E}_{0}=15 \mathrm{keV}\right)$ :Holes in subsurface Ag layer influencing the $\delta_{\mathrm{B}}$ yield of the glass overlayer (see: specimen. cross-section). 